\title{
GLOBAL ASYMPTOTIC STABILITY IN A PERIODIC LOTKA-VOLTERRA SYSTEM
}

\author{
K. GOPALSAMY
}

(Received 28 February 1984)

\begin{abstract}
A set of easily verifiable sufficient conditions are obtained for the existence of a globally asymptotically stable periodic solution in a Lotka-Volterra system with periodic coefficients.
\end{abstract}

\section{Introduction}

The purpose of this article is to derive a set of "easily verifiable" sufficient conditions for the existence of a globally asymptotically stable strictly positive (componentwise) periodic solution of the Lotka-Volterra system

$$
\frac{d x_{i}}{d t}=x_{i}\left\{b_{i}(t)-\sum_{j=1}^{n} a_{i j}(t) x_{j}(t)\right\} ; \quad t>0, n \geqslant 1 ; i=1,2, \ldots, n,
$$

where $b_{l}, a_{t j}(i, j=1,2, \ldots, n)$ are continuous positive periodic functions with a common period $\omega$. In ecology (1.1) denotes the dynamics of an $n$-species population system in which each individual competes with all others of the system for a common pool of resources. The assumption of periodicity of the parameters $b_{i}, a_{i j}$ in (1.1) is a way of incorporating periodicity of the environment (e.g. seasonal effects of weather, food supplies, mating habits etc.).

\footnotetext{
${ }^{1}$ School of Mathematical Sciences, The Flinders University of South Australian, Bedford Park, S. A. 5042.

(c) Copyright Australian Mathematical Society 1985, Serial-fee code 0334-2700/85
} 
We will need the following preparation; let $\mathbf{R}$ and $\mathbf{R}_{n}$ respectively denote the set of all real numbers and the $n$-dimensional real Euclidean space; $\mathbf{R}_{n}^{+}$will denote the nonnegative cone of $\mathbf{R}_{n}$. Define the constants $b_{i}^{l}, b_{i}^{u}, a_{i j}^{l}, a_{i j}^{u}(i, j=$ $1,2, \ldots, n)$ by the following

$$
\begin{aligned}
\inf _{t \in \mathbf{R}} b_{i}(t) & =\min _{t \in[0, \omega]} b_{i}(t)=b_{i}^{l}, \\
\inf _{t \in \mathbf{R}} a_{i j}(t) & =\min _{t \in[0, \omega]} a_{i j}(t)=a_{i j}^{l}, \\
\sup _{t \in \mathbf{R}} b_{i}(t) & =\max _{t \in[0, \omega]} b_{i}(t)=b_{i}^{u}, \\
\sup _{t \in \mathbf{R}} a_{i j}(t) & =\max _{t \in[0, \omega]} a_{t j}(t)=a_{i j}^{u} .
\end{aligned}
$$

We analyse the system (1.1) under the following assumption on the coefficients of (1.1);

$$
\begin{aligned}
& b_{i}^{\prime}>0 \text { and } a_{i i}^{\prime}>0 ; \quad i=1,2, \ldots, n . \\
& b_{i}^{\prime}>\sum_{\substack{j=1 \\
j \neq i}}^{n} a_{i j}^{u}\left(b_{j}^{u} / a_{\jmath j}^{\prime}\right) ; \quad i=1,2, \ldots, n .
\end{aligned}
$$

Since solutions of (1.1) corresponding to nonnegative initial conditions remain nonnegative it will follow that

$$
\frac{d x_{i}}{d t} \leqslant x_{i}\left\{b_{i}^{u}-a_{1 i}^{l} x_{i}\right\} ; \quad t>0, i=1,2, \ldots, n,
$$

as a consequence of which we have

$$
0<x_{i}(0) \leqslant b_{i}^{u} / a_{i t}^{l}=x_{i}^{u} \Rightarrow x_{i}(t) \leqslant b_{i}^{u} / a_{i i}^{l} ; \quad t>0, i=1,2, \ldots, n .
$$

Expressions (1.1) and (1.5) together lead to

$$
\frac{d x_{i}}{d t} \geqslant x_{i}\left\{b_{i}^{l}-\sum_{\substack{j=1 \\ j \neq 1}}^{n} a_{i j}^{u}\left(b_{j}^{u} / a_{j j}^{l}\right)-a_{i i}^{u} x_{i}\right\}, \quad t>0, i=1,2, \ldots, n .
$$

Now (1.2), (1.3) and (1.6) lead to

$$
\begin{aligned}
x_{i}(0) & \geqslant b_{i}^{\prime}-\sum_{\substack{j=1 \\
j \neq i}}^{n} a_{i j}^{u}\left(b_{j}^{u} / a_{j j}^{l}\right)=x_{i}^{\prime} \\
& \Rightarrow x_{i}(t) \geqslant x_{i}^{\prime} \quad \text { for } t \geqslant 0, i=1,2, \ldots, n .
\end{aligned}
$$


From the foregoing preparation we have the following:

Lemma. Assume that $b_{i}, a_{i j}(i, j=1,2, \ldots, n)$ are strictly positive continuous periodic functions with a common period $\omega>0$ such that (1.2) and (1.3) hold. Then the set $S$ defined by

$$
S=\left\{x=\left(x_{1}, \ldots, x_{n}\right) \in \mathbf{R}_{n}^{+} \mid x_{i}^{l} \leqslant x_{i} \leqslant x_{i}^{u} ; i=1,2, \ldots, n\right\}
$$

is invariant with respoct to (1.1).

\section{Existence of a periodic solution}

Let us consider the system (1.1) in $\mathbf{R}_{n}$ with the norm in $\mathbf{R}_{n}$ being defined by $\|x\|=\max _{1 \leqslant i \leqslant n}\left|x_{t}\right|, x=\left(x_{1}, x_{2}, \ldots, x_{n}\right) \in \mathbf{R}_{n}$. We know from the form of the systems (1.1) and (1.4) that there exists (for all finite values of $t$ ) a unique solution of (1.1) corresponding to any $x^{0}=\left(x_{1}^{0}, \ldots, x_{n}^{0}\right) \in \mathbf{R}_{n}$; let such a solution be denoted by

$$
\begin{aligned}
& x\left(t, x^{0}\right)=\left\{x_{1}\left(t, x^{0}\right), \ldots, x_{n}\left(t, x^{0}\right)\right\} ; \quad t>0, \\
& x\left(0, x^{0}\right)=x^{0} .
\end{aligned}
$$

We define a shift operator also known as a Poincaré period map $A: \mathbf{R}^{n} \rightarrow \mathbf{R}^{n}$ by the formula

$$
A x^{0}=x\left(\omega, x^{0}\right), \quad x^{0} \in \mathbf{R}_{n} .
$$

If one can show that the operator $A$ has a fixed point say $z=\left(z_{1}, z_{2}, \ldots, z_{n}\right)$, then it will follow that for the system (1.1) there is a solution say $x^{*}(t)$ defined for $t \in[0, \omega]$ satisfying the condition

$$
x^{*}(\omega)=x^{*}(0)=z
$$

or equivalently

$$
x_{i}^{*}(\omega, z)=z_{i} ; \quad i=1,2, \ldots, n .
$$

Since the right side of (1.1) is periodic in $t$ for fixed $x \in \mathbf{R}_{n}$, it will follow that $x^{*}(t)$ can be extended for $t>\omega$ by periodicity, in the sense that

$$
x^{*}(m \omega+t)=x^{*}(t) \text { for } m=1,2,3, \ldots,
$$

for all $t \geqslant 0$, and such an extension will be a periodic solution of (1.1). Thus the existence of periodic solutions of (1.1) will follow from the existence of fixed points of the shift operator $A$ defined above in (2.2).

The following result is well known:

THEOREM (Brouwer). Suppose that a continuous operator $U$ maps a closed bounded convex set $\Omega \subset \mathbf{R}_{n}$ into itself. Then $\Omega$ contains at least one $y \in \Omega$ for which $U y=y$ holds. 
THEOREM 2.1. Suppose the coefficients of (1.1) satisfy (1.2) and (1.3). Then (1.1) has at least one strictly positive periodic solution.

Proof. Define a set $\Omega \subset \mathbf{R}_{n}$ as follows:

$$
\Omega=\left\{x=\left(x_{1}, \ldots, x_{n}\right) \in \mathbf{R}_{n} \mid \min _{1 \leqslant i \leqslant n} x_{i}^{\prime} \leqslant x_{j} \leqslant \max _{1 \leqslant i \leqslant n} x_{i}^{u} ; j=1,2, \ldots, n\right\} .
$$

The set $\Omega$ is a bounded, closed and convex set in $\mathbf{R}_{n}$ and the operator $A$ maps $\Omega$ into itself since $\Omega$ is invariant with respect to (1.1). This means that $x^{0}=\left(x_{1}^{0}, \ldots, x_{n}^{0}\right) \in \Omega \Rightarrow\left\{x_{1}\left(t, x^{0}\right), x_{2}\left(t, x^{0}\right), \ldots, x_{n}\left(t, x^{0}\right)\right\} \in \Omega$

$$
\text { for all } t \geqslant 0
$$

and hence $\left\{x_{1}\left(\omega, x^{0}\right), \ldots, x_{n}\left(\omega, x^{0}\right)\right\} \in \Omega$ which implies that $A \Omega \subset \Omega$. The solutions of (1.1) are continuous functions of their initial values, from which the continuity of the operator follows. Now by the Brouwer theorem the existence of at least one fixed point of $A$ in $\Omega$ follows. Since such a fixed point has positive co-ordinates, the corresponding periodic solution is strictly positive by the invariance of $\Omega$, and the proof is complete.

\section{Global asymptotic stability}

By definition we say that a periodic solution, say $u(t)=\left(u_{1}(t), \ldots, u_{n}(t)\right)$, of (1.1) is globally asymptotically stable (or attractive) if and only if every other solution $x(t)=\left\{x_{1}(t), \ldots, x_{n}(t)\right\}$ of $(1.1)$ with $x_{i}(0)>0(i=1,2, \ldots, n)$ is defined for all $t \geqslant 0$ and satisfies

$$
\lim _{t \rightarrow \infty}\left|u_{i}(t)-x_{i}(t)\right|=0 ; \quad i=1,2, \ldots, n .
$$

A consequence of such a global asymptotic stability is that there cannot be another strictly positive periodic solution of (1.1)

THEOREM 3.1. Suppose the coefficients of (1.1) satisfy (1.2), (1.3) and the following

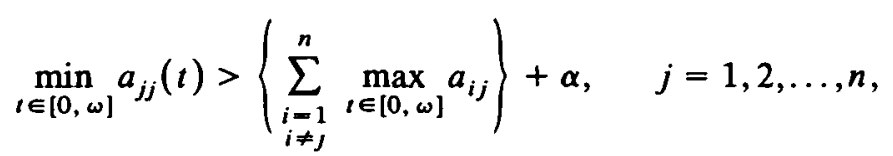

for some positive number $\alpha$. Then (1.1) has a periodic solution (with strictly positive components) which is globally asymptotically stable.

Proof. Let $x(t)=\left\{x_{1}(t), \ldots, x_{n}(t)\right\}$ be any solution of $(1.1)$ with $x_{i}(0)>0$ $(i=1,2, \ldots, n)$. Since solutions of (1.1) remain nonnegative we can let

$$
U_{i}(t)=\log u_{i}(t) \text { and } X_{i}(t)=\log x_{i}(t)
$$


where $u(t)=\left\{u_{1}(t), \ldots, u_{n}(t)\right\}$ is a strictly positive periodic solution of (1.1), the existence of which follows by Theorem 2.1. It will follow from (3.3) and (1.1) that

$$
\begin{array}{r}
\frac{d}{d t}\left[U_{i}(t)-X_{i}(t)\right]=-a_{i i}(t)\left[e^{U_{i}(t)}-e^{X_{i}(t)}\right]-\sum_{\substack{j=1 \\
j \neq i}}^{n} a_{i j}(t)\left[e^{U_{j}(t)}-e^{X_{j}(t)}\right], \\
i=1,2, \ldots, n ; t>0 .
\end{array}
$$

Consider a Lyapunov function $v(t)$ defined by

$$
v(t)=\sum_{i=1}^{n}\left|U_{i}(t)-X_{i}(t)\right| ; \quad t \geqslant 0 .
$$

Calculating the upper right derivative $D^{+} v$ of $v$ along the solutions of (3.4), we have

$$
\begin{aligned}
D^{+} v(t) & \leqslant \varepsilon \sum_{i=1}^{n}\left[\left|a_{i i}(t) \| e^{U_{i}}-e^{X_{i}}\right|-\sum_{\substack{j=1 \\
j \neq 1}}^{n}\left|a_{i j}(t)\right| \mid e^{U_{J}}-e^{X_{J} \mid}\right] \\
& \left.\leqslant-\sum_{i=1}^{n}\left[\left|a_{i i}(t)\right|-\sum_{\substack{j=1 \\
j \neq i}}^{n}\left|a_{j i}(t)\right|\right] \mid e^{U_{i}}-e^{X_{i} \mid}\right] \\
& \leqslant-\alpha \sum_{i=1}^{n}\left|u_{i}(t)-x_{i}(t)\right| .
\end{aligned}
$$

An integration of (3.6) leads to

$$
\sum_{i=1}^{n}\left|U_{t}(t)-X_{i}(t)\right|+\alpha \int_{0}^{t} \sum_{i=1}^{n}\left|u_{i}(s)-x_{i}(s)\right| d s \leqslant v(0)<\infty .
$$

It follows from (3.7) that $\sum_{i-1}^{n}\left|U_{t}(t)-X_{t}(t)\right|$ is bounded for $t \geqslant 0$, implying that the right side of (3.4) remains bounded for $t \geqslant 0$; thus it will follow then that $\sum_{i=1}^{n}\left|U_{t}(t)-X_{i}(t)\right|$ is uniformly continuous on $[0, \infty)$. Consequently the uniform continuity of $\sum_{i-1}^{n}\left|u_{i}(t)-x_{i}(t)\right|$ on $[0, \infty)$ will follow. Such a uniform continuity with the integrability on $[0, \infty)$ (see (3.7)) of $\sum_{i=1}^{n}\left|u_{i}(t)-x_{i}(t)\right|$ will lead to (3.1) (for more details see [3]) and the proof is complete.

\section{An autonomous system}

We will briefly indicate a study of autonomous systems of the form

$$
\frac{d y_{i}}{d t}=y_{i}\left\{\beta_{t}-\sum_{j=1}^{n} \alpha_{i j} y_{j}\right\} ; \quad t>0, i=1,2, \ldots, n,
$$


where $\beta_{i}, \alpha_{i j}(i, j=1,2, \ldots, n)$ are positive constants. For autonomous systems of the form (4.1), the existence of at least one positive (componentwise) steady state is a necessary condition for the "persistence" of the system (4.1); by persistence we mean here that no species of the system (4.1) is threatened with extinction, which is translated into a mathematical condition of the form $\lim _{t \rightarrow \infty}$ inf $x_{i}(t)>0(i=1,2, \ldots, n)$. We refer to [3] for more details regarding persistence and existence of steady states. For (4.1) we have the following:

TheORem 4.1. Assume the constant parameters $\beta_{t}, \alpha_{i j}(i, j=1,2, \ldots, n)$ satisfy the following

(i) $\alpha_{\jmath j}>0$ and $\alpha_{j j}>\left(\sum_{i=1 ; 1 \neq j}^{n} \alpha_{i j}\right)+\varepsilon, j=1,2, \ldots, n$,

for some positive number $\varepsilon$.

(ii) $\beta_{i}>\sum_{j=1}^{n} \alpha_{i j}\left(\beta_{j} / \alpha_{j j}\right), i=1,2, \ldots, n$.

Then there exists a unique steady state $y^{*}=\left(y_{1}^{*}, \ldots, y_{n}^{*}\right)$ with $y_{j}^{*}>0(j=$ $1,2, \ldots, n)$ of $(4.1)$ satisfying

$$
\sum_{j=1}^{n} \alpha_{i j} y_{j}^{*}=\beta_{i} ; \quad i=1,2, \ldots, n,
$$

and the steady state $y^{*}$ of (4.1) is globally asymptotically stable, in the sense that any arbitrary solution $y(t)=\left\{y_{1}(t), \ldots, y_{n}(t)\right\}$ of $(4.1)$ with $y_{j}(0)>0(j=1,2, \ldots, n)$ satisfies the condition

$$
\lim _{t \rightarrow \infty} y_{i}(t)=y_{i}^{*} ; \quad i=1,2, \ldots, n .
$$

Proof. Any nonzero steady state of (4.1) is a solution of the linear system of equations

$$
\sum_{j=1}^{n} \alpha_{i j} y_{j}=\beta_{i}, \quad i=1,2, \ldots, n .
$$

One can also see that any solution of (4.6) is also a fixed point of the mapping $T$ defined by $T: D \rightarrow \mathbf{R}_{n}$ where

$$
\begin{aligned}
& T(y)=\left\{T_{1}(y), \ldots, T_{n}(y)\right\}, \\
& T_{i}(y)=y_{i}-\left(\alpha_{i i} / \sigma_{i i}\right) y_{i}+\left[\beta_{i}-\sum_{\substack{j=1 \\
j \neq i}}^{n} \alpha_{i j} y_{j}\right] / \sigma_{i i}, \\
& \sigma_{i i}=\alpha_{i i}+\sum_{j=1}^{n} \alpha_{i j}\left(\beta_{j} / \alpha_{j j}\right), \quad i=1,2, \ldots, n, \\
& D=\left\{y=\left(y_{1}, \ldots, y_{n}\right) \in \mathbf{R}_{n} \mid 0 \leqslant y_{i} \leqslant \beta_{l} / \alpha_{i i}\right\}, \quad i=1,2, \ldots, n .
\end{aligned}
$$


It is not difficult to verify using (4.2) and (4.3) that any fixed point of $T$ has to be in $D$, and $T$ is a contraction on $D$. The existence of a unique fixed point of $T$, say $y^{*}$, will follow and one can directly verify that no component of $y^{*}$ can be zero or negative by (4.3).

The global asymptotic stability of $y^{*}$ is achieved exactly in the same manner as in Theorem 3.1 by substituting

$$
U_{i}(t) \equiv \log y_{i}^{*} \quad \text { and } \quad X_{i}(t)=\log y_{i}(t), \quad i=1,2, \ldots, n,
$$

and repeating the remainder of the proof of that theorem and we do not go into such a repetition.

\section{Some comments}

The results obtained here provide a significant generalisation of the author's earlier result [2] where the parameters $a_{\imath}$ in (1.1) were assumed to be time invariant. Periodic Lotka-Volterra systems of the form (1.1) have been previously considered by Cushing [1] under different and weaker assumptions; locally asymptotic stability of periodic solutions have been established by Cushing [1] after proving the existence of periodic solutions by means of bifurcation theoretic techniques; the stability condition of Cushing [1] requires a knowledge of the periodic solution. The major implication of our result in Theorem 3.1 is that under the conditions of that theorem, no complex behaviour of the system (1.1) is possible. For some remarks regarding the evolutionary and ecological significance of the periodicity of the environment, we refer to [4]. By following the method of [4], one can further generalise the result of Theorem 3.1 to periodic systems with time delays in the interspecific interactions.

\section{References}

[1] J. M. Cushing, "Stable limit cycles in time dependent multispecies interactions", Math. Biosci. 31 (1976), 259-273.

[2] K. Gopalsamy, "Exchange of equilibria in two species Lotka-Volterra competition models", $J$. A ustral. Math. Soc. Ser. B 24 (1982), 160-170.

[3] K. Gopalsamy, "Global asymptotic stability in Volterra's population systems", J. Math. Biol. 19 (1984), 157-168.

[4] K. Gopalsamy, "Harmless delays in a periodic ecosystem", J. Austral. Math. Soc. Ser. B 25 (1984), 349-365. 